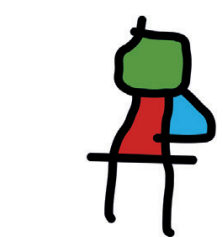

SEICAIP

\section{Allergologia et immunopathologia}

Sociedad Española de Inmunología Clínica, Alergología y Asma Pediátrica

wWw.all-imm.com

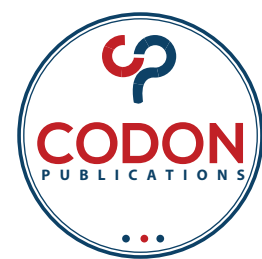

OPEN ACCESS (c)

RESEARCH ARTICLE

\title{
KCNQ10T1 promotes the proliferation and migration of psoriatic keratinocytes by regulating miR-183-3p/GAB1
}

\author{
Ting Liu*, Xi Duan, Jia He, Chuan Yang
}

Department of Dermatology, Affiliated Hospital of North Sichuan Medical College, Nanchong City 637000, Sichuan Province, China

Received 15 July 2021; Accepted 11 August 2021

Available online 1 September 2021

\section{KEYWORDS \\ GAB1; \\ KCNQ10T1; \\ migration; \\ miR-183-3p; \\ proliferation; \\ psoriasis}

\begin{abstract}
Background: Differentially expressed long non-coding RNAs (IncRNA) have been reported to be involved in the proliferation and migration of keratinocyte. Potassium voltage-gated channel subfamily Q member 1 overlapping transcript 1 (KCNQ1OT1) was implicated in the pathogenesis of various diseases, including cancer, sepsis, diabetic cardiomyopathy, and atherosclerosis. In this study, the influence of KCNQ10T1 on the proliferation and migration of psoriatic keratinocytes was explained.

Methods: Cultured human keratinocyte cell line ( $\mathrm{HaCaT})$ was incubated with tumor necrosis factor- $\alpha($ TNF- $\alpha)$. Cell viability and migration were assessed by MTT assay and wound healing, respectively. Target miRNA of KCNQ10T1 was identified by luciferase activity and RNA immunoprecipitation (RIP) assays.

Results: KCNQ10T1 was up-regulated in TNF- $\alpha$-treated HaCaT cell line, and knockdown of KCNQ10T1 reduced viability and suppressed the migration of TNF- $\alpha$-treated HaCaT cell line. KCNQ10T1 was bound to microRNA-183-3p (miR-183-3p) and negatively regulated its expression. Over-expression of growth factor receptor binding 2-associated binding protein 1 (GAB1) counteracted with the suppressive effects of KCNQ10T1-induced silence on the viability and migration of TNF- $\alpha$-treated HaCaT cells.

Conclusion: KCNQ10T1 silence suppressed the proliferation and migration of TNF- $\alpha$-treated $\mathrm{HaCaT}$ cells through regulation of miR-183-3p/GAB1.

(c) 2021 Codon Publications. Published by Codon Publications.
\end{abstract}

*Corresponding author: Ting Liu, Department of Dermatology, Affiliated Hospital of North Sichuan Medical College, No. 1, Maoyuan South Road, Shunqing District, Nanchong City 637000, Sichuan Province, China. E-mail address: liuting_666@163.com 


\section{Introduction}

Psoriasis, a papulosquamous, relapsing, and chronic dermatitis, affects about $1-3 \%$ of global population, with increasing incidences. ${ }^{1}$ Although the pathogenesis and etiology of psoriasis are complicated, genetic, immune, environmental, and psychological factors are regarded as key regulators in its pathogenesis. ${ }^{2}$ Inflammatory cell infiltration, neovascularization, keratinosis, and keratinocyte dysplasia are the pathological alterations of psoriasis. ${ }^{3}$ Keratinocytes, predominant cell types of the epidermis, form barrier against environmental damages. ${ }^{4}$ Abnormal migration and hyperproliferation of keratinocytes are implicated in the pathogenesis of chronic inflammatory skin diseases, such as psoriasis. ${ }^{5}$ Therefore, suppression of psoriatic keratinocyte proliferation and migration ameliorated psoriatic lesional microenvironment and attenuated psoriasis. ${ }^{6}$

Long non-coding RNAs (IncRNAs) are differentially expressed in psoriatic skin compared to the healthy skin, ${ }^{7}$ and IncRNAs are regarded as diagnostic biomarkers and therapeutic targets in psoriasis. ${ }^{8}$ For example, IncRNA WAKMAR1 enhanced the migration of keratinocyte to regulate skin wound healing. ${ }^{9}$ MEG3, long non-coding RNA, promoted cell apoptosis and repressed proliferation of activated cultured human keratinocyte cell line (HaCaT). ${ }^{10}$ LncRNA potassium voltage-gated channel subfamily $Q$ member 1 overlapping transcript 1 (KCNQ10T1) has been reported to be involved in the pathogenesis of various diseases. KCNQ10T1 ameliorated anesthesia-induced nerve injury, ${ }^{11}$ and promoted osteogenic differentiation to attenuate osteolysis. ${ }^{12}$ KCNQ10T1 also regulated cancer cell proliferation, apoptosis, and metastasis. ${ }^{13-15}$ Lipopolysaccharide-induced inflammatory response in mice was reduced by silence of KCNQ10T1, ${ }^{16}$ and the fracture healing was accelerated by KCNQ10T1. ${ }^{17}$ However, the role of KCNQ10T1 in psoriasis remains unclear.

LncRNAs and microRNAs (miRNAs) are widely involved in competitive endogenous RNA (ceRNA) model, where IncRNAs act as sponges for miRNAs and play a role in disease. ${ }^{18}$ MiR-26a-5p interacted with KCNQ10T1 to mediate myocardial infarction. ${ }^{19}$ KCNQ10T1-microRNA-183-3p (miR183-3p) axis was involved in proliferation and apoptosis of vascular smooth muscle cells. ${ }^{20}$ Since miR-183-3p has been reported to repress the migration and proliferation of keratinocytes in psoriasis, ${ }^{21}$ KCNQ10T1 was hypothesized to participate in migration and proliferation of keratinocytes through regulation of miR-183-3p.

In this study, the effects of KCNQ10T1 on the proliferation and migration of tumor necrosis factor- $\alpha$ (TNF$\alpha$ )-treated $\mathrm{HaCaT}$ cell line were investigated, with identification of the underlying mechanism.

\section{Materials and methods}

\section{Cell culture and treatment}

$\mathrm{HaCaT}$ (Chinese Academy of Sciences, Shanghai, China) cell line was maintained in Eagle's Minimum Essential medium (MEM; Lonza, Basel, Switzerland) with streptomycinpenicillin and $10 \%$ newborn calf serum (Lonza). Cells were seeded in a culture dish for $24 \mathrm{~h}$, then incubated with
10-ng/mL TNF- $\alpha$ (Sigma Aldrich, St. Louis, MO, USA) for another $24 \mathrm{~h}$ for functional assays.

\section{Enzyme-linked-immunosorbent serologic assay (ELISA)}

The culture medium of $\mathrm{HaCaT}$ was harvested, and the levels of TNF- $\alpha$, IL- $1 \beta$, and IL- 6 were detected by commercial kits (Sigma Aldrich).

\section{Cell viability and wound healing}

$\mathrm{HaCaT}$ cell line $\left(1 \times 10^{4}\right.$ cells/well) was incubated with TNF- $\alpha$, and transfected with si-KCNQ10T1 (\#1 and \#2) or the negative control (si-NC; 5'-CACGATAAGACAATGTATTT-3') (GenePharma, Suzhou, Jiangsu Province, China) by Lipofectamine 2000 (Sigma Aldrich). si-KCNQ10T1 \#1 and pcDNA-growth factor receptor binding 2-associated binding protein 1 (GAB1) (GenePharma) co-transfected into $\mathrm{HaCaT}$ cells. After $48 \mathrm{~h}$, cells were seeded for another 24,48 , or $72 \mathrm{~h}$, treated with 3-(4,5-dimethylthiazol-2-yl)-2,5-diphenyltetrazolium bromide (MTT) reagent (Sigma Aldrich) for $4 \mathrm{~h}$, and incubated with $100-\mu \mathrm{L}$ dimethyl sulfoxide (DMSO). Absorbance at $490 \mathrm{~nm}$ was measured with microplate autoreader (Thermo Fisher, Waltham, MA, USA). For cell migration assay, HaCaT cells $\left(1 \times 10^{4}\right.$ cells/well) with indicated treatment and transfection were seeded and scratched. After $24 \mathrm{~h}$, cells were photographed and the wound area was calculated.

\section{Dual luciferase reporter and RNA immunoprecipi- tation (RIP) assays}

The potential binding target of KCNQ10T1 was predicted by DIANA Tools prediction (http://diana.imis.athena-innovation.gr/DianaTools/index.php). Sequences of wildtype or mutant KCNQ10T1 were subcloned into pmirGLO luciferase reporter vector (Promega, Madison, WI, USA), and named as KCNQ10T1-wt or KCNQ10T1-mut. HaCaT cells $\left(3 \times 10^{4}\right.$ cells/ well) were seeded and co-transfected KCNQ10T1-wt or KCNQ10T1-mut with miR-183-3p mimics or the negative control (NC mimics; 5'-UCUACUCUUUCUAGGAGGUUGUGA-3'). After $48 \mathrm{~h}$, the luciferase activities were determined by Lucifer Reporter Assay System (Promega). For RIP assay, HaCaT cells were transfected with pcDNA-KCNQ10T1 (GenePharma) for $48 \mathrm{~h}$, and lysed by Magna RIP Kit (EMD Millipore, Billerica, MA, USA). The lysates were incubated with protein G Sepharose beads coated with anti-lgG or anti-AGO2 antibodies (Abcam, Cambridge, MA, USA). The beads were harvested and RNAs were isolated for quantitative reverse transcription polymerase chain reaction (qRT-PCR) analysis.

\section{qRT-PCR}

RNAs were isolated from $\mathrm{HaCaT}$ cells using Trizol (Invitrogen, Carlsbad, CA, USA), and reverse-transcribed into complementary DNAs (cDNA). The cDNAs were conducted with SYBR Green Master (Roche, Mannheim, Germany) for qRT-PCR analysis of KCNQ10T1 and miR-183-3p. GAPDH or U6 was used as an endogenous control. The used primer sequences are as follows: GAPDH (forward: 
5'-ACCACAGTCCATGCCATCAC-3'; reverse: 5'-CCACCACCC TGTTGCTGTA-3'), KCNQ10T1 (forward: 5'-GCACTCTGGGTCC TGTTCTC-3'; reverse: 5'-CACTTCCCTGCCTCCTACAC-3'), miR183-3p (forward: 5'-CGCGGTATGGCACTGGTAGA-3'; reverse: 5'-AGTGCAGGGTCCGAGGTATTC-3'), and U6 (forward: 5'-CTCGCTTCGGCAGCACA-3'; reverse: 5'-AACGCTTCACGAA TTTGCGT-3').

\section{Statistical analysis}

All the results were expressed as mean \pm SEM. The statistical analysis was performed by one-way analysis of variance or student's $t$-test; $\mathrm{P}<0.05$ was considered as statistically significant.

\section{Results}

\section{KCNQ10T1 contributed to the proliferation of keratinocytes}

$\mathrm{HaCaT}$ cell lines were incubated with TNF- $\alpha$ for instituting hyperproliferation in keratinocytes. KCNQ10T1 was up-regulated in TNF- $\alpha$-induced HaCaT cells (Figure 1A). TNF$\alpha$ treatment increased $\mathrm{HaCaT}$ cells viability (Figure $1 \mathrm{~B}$ ).
TNF- $\alpha$-treated $\mathrm{HaCaT}$ cells were transfected with si-KCNQ10T1 \#1 and \#2 for the knockdown of KCNQ10T1 (Figure 1A). Knockdown of KCNQ10T1 reduced the TNF- $\alpha$-induced HaCaT cells viability (Figure 1B), suggesting that KCNQ10T1 was involved in TNF- $\alpha$-mediated $\mathrm{HaCaT}$ cells proliferation. Moreover, the levels of TNF- $\alpha$, IL- $1 \beta$, and IL- 6 in HaCaT cells were increased post-TNF- $\alpha$ treatment (Supplemental Figure S1), and knockdown of KCNQ10T1 reduced TNF- $\alpha$-induced levels of TNF- $\alpha$, IL-1 $\beta$, and IL-6 in HaCaT cells (Figure S1).

\section{KCNQ10T1 contributed to the migration of keratinocytes}

TNF- $\alpha$ treatment also induced the migration of HaCaT cells (Figure 2). However, knockdown of KCNQ10T1 suppressed TNF- $\alpha$-induced $\mathrm{HaCaT}$ cells migration, suggesting the anti-migratory effect of KCNQ10T1-induced silence against TNF- $\alpha$-induced hyperproliferation in keratinocytes.

\section{KCNQ10T1 negatively regulated the expression of miR-183-3p}

DIANA Tools prediction demonstrated that KCNQ10T1 contains potential binding sites of miR-183-3p (Figure 3A).
(A)

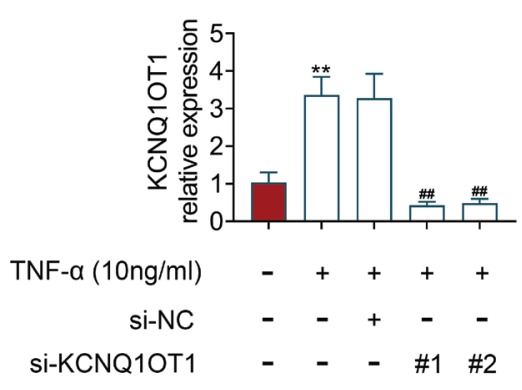

(B)

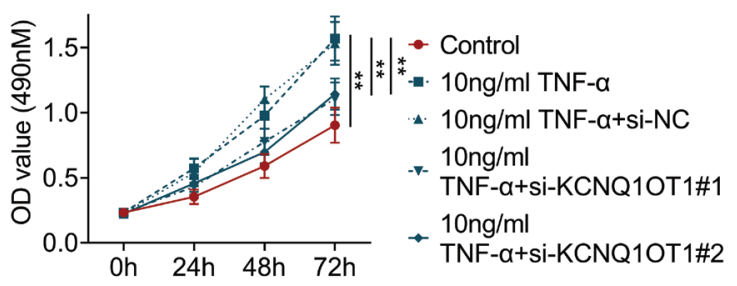

Figure 1 KCNQ10T1 contributed to the proliferation of keratinocytes. (A) KCNQ10T1 was up-regulated in TNF- $\alpha$-treated HaCaT cells, and transfection with si-KCNQ10T1 \#1 and \#2 reduced KCNQ10T1 expression in TNF- $\alpha$-treated HaCaT cells. (B) Knockdown of KCNQ10T1 reduced the viability of TNF- $\alpha$-induced $\mathrm{HaCaT}$ cells. ${ }^{* *} \mathrm{P}<0.01$ vs. control, ${ }^{\# \# P}<0.01 \mathrm{vs} .10 \mathrm{ng} / \mathrm{mL} \mathrm{TNF}-\alpha$.
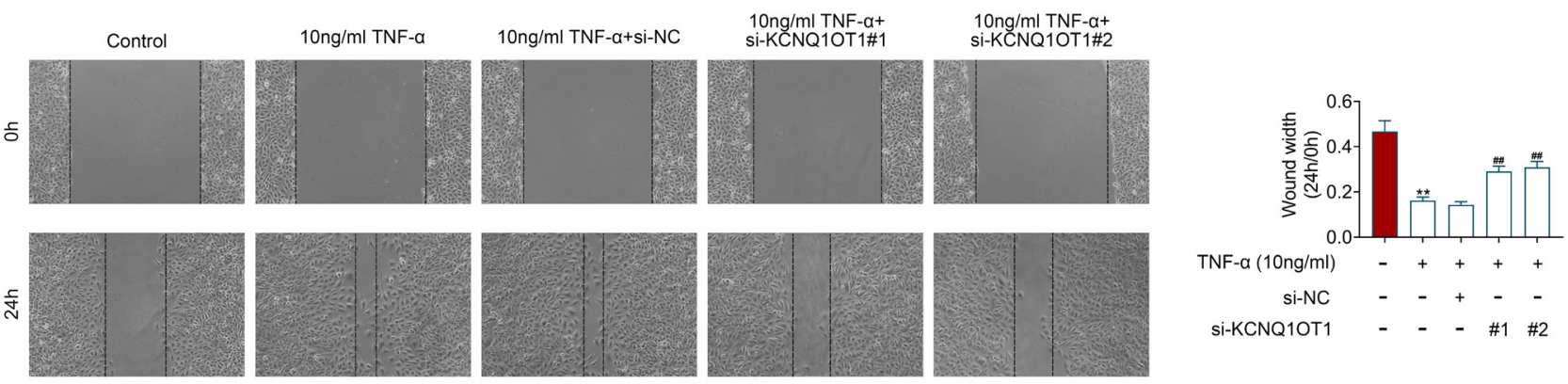

Figure 2 KCNQ10T1 contributed to the migration of keratinocytes. Knockdown of KCNQ10T1 reduced the migration of TNF- $\alpha$ treated $\mathrm{HaCaT}$ cells. ${ }^{* *} \mathrm{P}<0.01$ vs. control, ${ }^{\#} \mathrm{P}<0.01$ vs. $10 \mathrm{ng} / \mathrm{mL}$ TNF- $\alpha$. 
(A)

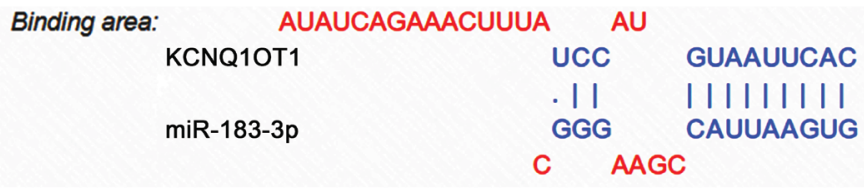

(B)

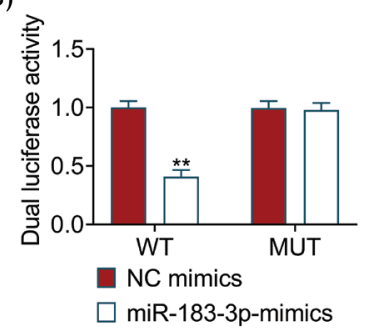

(C)

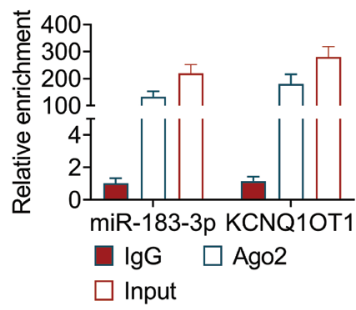

(D)

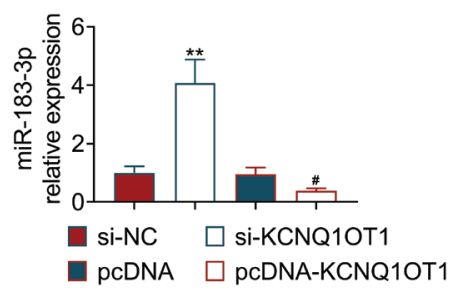

Figure 3 KCNQ10T1 inhibited the expression of miR-183-3p. (A) The potential binding sites between KCNQ10T1 and miR-183-3p were predicted by DIANA Tools. (B) Transfection of miR-183-3p mimics reduced the luciferase activity of KCNQ10T1-wt, while it had no effect on KCNQ10T1-mut. (C) Enrichment of KCNQ10T1 and miR-183-3p in HaCaT cells after over-expression of KCNQ10T1. (D) Knockdown of KCNQ10T1 increased the expression of miR-183-3p in HaCaT cell, while over-expression of KCNQ10T1 decreased the expression of miR-183-3p. " $\mathrm{P}<0.01$ vs. NC mimics or si-NC. ${ }^{\#} \mathrm{P}<0.05$ vs. pCDNA.

(A)

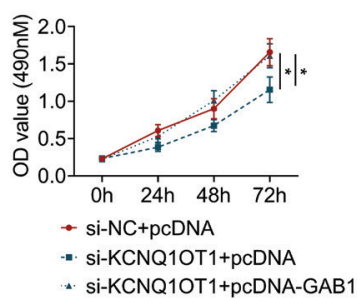

(B)

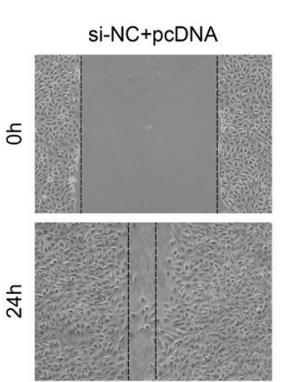

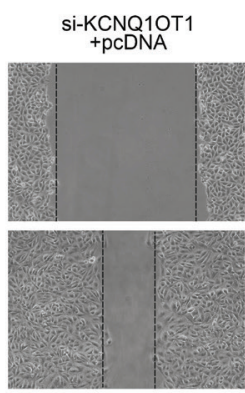

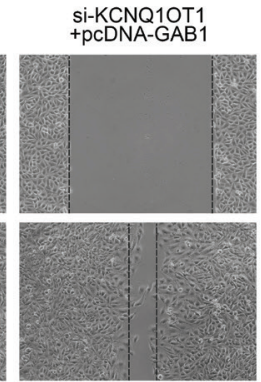

Figure 4 KCNQ10T1 contributed to the proliferation and migration of keratinocytes through regulation of miR-183-3p. (A) KCNQ10T1 silence-induced reduction in the viability of TNF- $\alpha$-treated HaCaT cells was restored by the over-expression of GAB1. (B) Over-expression of GAB1 attenuated KCNQ10T1 silence-induced reduction in the migration of TNF- $\alpha$-treated HaCaT cells. ${ }^{*} \mathrm{P}<0.05,{ }^{* *} \mathrm{P}<0.01$ vs. si-NC $+\mathrm{pCDNA},{ }^{\# \# P}<0.01$ vs. si-KCNQ10T1 + pcDNA.

Transfection with miR-183-3p mimics reduced the luciferase activity of KCNQ10T1-wt, while it had no significant effects on KCNQ10T1-mut (Figure 3B), demonstrating the binding relationship between KCNQ10T1 and miR-183-3p. Moreover, data from RIP assay depicted the enrichment of KCNQ10T1 and miR-183-3p in $\mathrm{HaCaT}$ cells after over-expression of KCNQ10T1 (Figure 3C), confirming that KCNQ10T1 was bound to miR-183-3p. KCNQ10T1 also negatively regulated the expression of miR-183-3p in HaCaT cell (Figure 3D), indicating that KCNQ10T1 might be a ceRNA of miR-183-3p in TNF- $\alpha$-induced hyperproliferation in keratinocytes.

\section{KCNQ10T1 contributed to the proliferation and migration of keratinocytes through regulation of miR-183-3p-GAB1 axis}

In order to investigate the role of KCNQ10T1/miR-183-3p/ GAB1 in the progress of psoriasis, TNF- $\alpha$-treated $\mathrm{HaCaT}$ cell line was co-transfected with si-KCNQ10T1 \#1 and pCDNA-GAB1. Silence of KCNQ10T1-induced reduction in the viability of TNF- $\alpha$-treated $\mathrm{HaCaT}$ cell line was restored by the over-expression of GAB1 (Figure 4A). Over-expression of GAB1 also attenuated KCNQ10T1 silence-induced decrease in the migration of TNF- $\alpha$-treated HaCaT cells (Figure 4B), revealing that KCNQ10T1 silence suppressed the proliferation and migration of keratinocytes through regulation of miR-183-3p-GAB1 axis.

\section{Discussion}

Emerging evidence has shown that IncRNAs were dysregulated in patients with psoriasis, and IncRNAs function as ceRNAs to sponge miRNAs during the regulation of keratinocyte proliferation and disturbed differentiation. ${ }^{8}$ Result of this study identified that a novel IncRNA, KCNQ10T1, was implicated in the pathogenesis of psoriasis. 

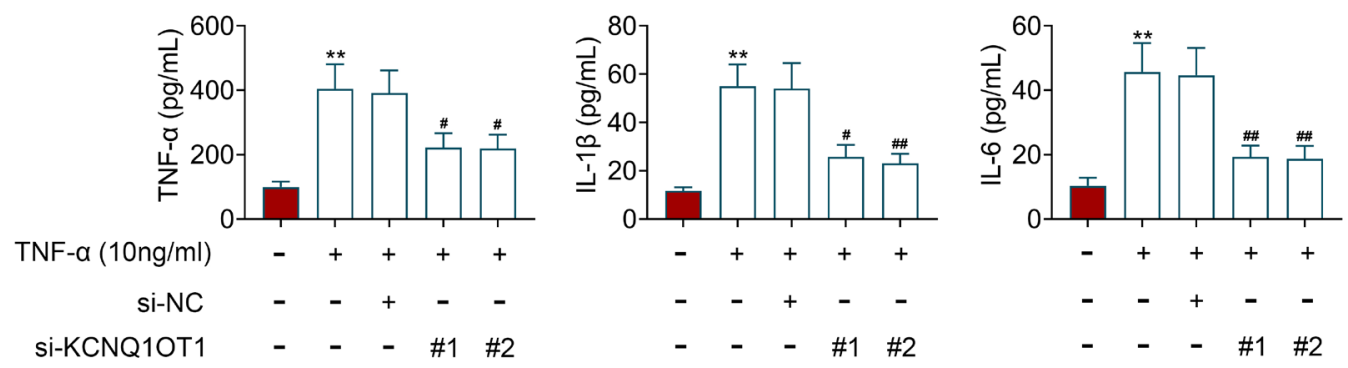

Figure S1 TNF- $\alpha$ treatment increased the levels of TNF- $\alpha$, IL-1 $\beta$, and IL- 6 in HaCaT cells, whereas knockdown of KCNQ10T1 reduced the levels of TNF- $\alpha$, IL- $1 \beta$, and IL-6 in TNF- $\alpha$-treated HaCaT cells. ${ }^{* *} \mathrm{P}<0.01$ vs. control. ${ }^{\#} \mathrm{P}<0.05,{ }^{\# \# P<0.01 ~ v s . ~} 10 \mathrm{ng} / \mathrm{mL}$ TNF- $\alpha$.

Previous study has shown that TNF- $\alpha$ is implicated in the pathogenesis of psoriasis by increasing the proliferation and migration of keratinocytes. ${ }^{22}$ Therefore, TNF- $\alpha$-treated $\mathrm{HaCaT}$ cell line was widely used as a cell model of psoriatic keratinocytes. ${ }^{22}$ Here, TNF- $\alpha$ treatment induced the up-regulation of KCNQ10T1 in HaCaT cells, suggesting that KCNQ10T1 might be involved in the development of psoriasis. However, the expression level of KCNQ10T1 in psoriatic lesional skin should be detected in the future research.

Hyperproliferation of keratinocytes mimics psoriasis lesional microenvironment through induction of infiltration of immunocytes and tacanthosis. ${ }^{23}$ Migration of keratinocytes recapitulates the psoriasis lesional microenvironment and stimulates wound re-epithelialization during the development of psoriasis. ${ }^{23}$ Suppression of migration and proliferation in keratinocytes facilitates the prevention of psoriasis. ${ }^{23}$ Proliferation and migration of bladder cancer cells have been reported to be promoted by KCNQ10T1. ${ }^{24}$ This study established that knockdown of KCNQ10T1 reduced cell viability and suppressed the migration of TNF$\alpha$-treated $\mathrm{HaCaT}$ cells, indicating the anti-proliferative and anti-migratory effects of KCNQ10T1-induced silence against psoriasis. Moreover, dysregulation of interplay between immune cells, keratinocytes, and skin-resident cells is a common pathogenesis of psoriasis. ${ }^{25}$ Inflammatory cytokines secreted by immune cells promoted the proliferation and migration of keratinocytes, ${ }^{26}$ and inhibition of inflammation is thus beneficial for psoriasis. ${ }^{26}$ Considering that KCNQ10T1 contributed to lipopolysaccharide-induced inflammatory response in mice, ${ }^{16}$ silence of KCNQ10T1 might exert anti-inflammatory effect against psoriasis.

MiR-183-3p was reported to suppress the proliferation and migration of keratinocytes, ${ }^{21}$ and KCNQ10T1 could bind with $\mathrm{miR}-183-3 p$ to regulate the proliferation and apoptosis of vascular smooth muscle cells. ${ }^{20}$ Data from luciferase activity and RIP assays revealed the direct binding relationship between KCNQ10T1 and miR-183-3p. Moreover, KCNQ10T1 negatively regulated the expression of miR183-3p in HaCaT cells, suggesting that KCNQ10T1 might regulate negatively miR-183-3p expression and thus contribute to the proliferation and migration of keratinocytes.

GAB1 was identified as a binding target of miR-183-3p, and was involved in the proliferation and migration of keratinocytes. ${ }^{21}$ Previous study has shown that GAB1 is required for the differentiation and growth of epithelium, and GAB1 regulates the migration of keratinocytes to participate in the development of psoriasis. ${ }^{27}$ Results of this study revealed that over-expression of GAB1 counteracted with the suppressive effects of KCNQ10T1-induced silence on the proliferation and migration of TNF- $\alpha$-treated $\mathrm{HaCaT}$ cells, thereby confirming that KCNQ10T1 contributed to psoriasis through regulation of miR-183-3p-GAB1 axis.

In summary, this was the first evidence demonstrating that KCNQ10T1 was up-regulated in TNF- $\alpha$-treated $\mathrm{HaCaT}$ cells. Knockdown of KCNQ10T1 reduced cell viability and suppressed the migration of TNF- $\alpha$-treated $\mathrm{HaCaT}$ cells through regulation of miR-183-3p-GAB1 axis. These results might provide a potential target for treating psoriasis. However, the effects of KCNQ10T1/miR-183-3p/GAB1 on the in vivo psoriatic model need further investigation.

\section{Competing interests}

The authors state that there are no conflicts of interest to disclose.

\section{Contribution of authors}

Ting Liu designed the study, and supervised the data collection; Xi Duan analyzed and interpreted the data; Jia He and Chuan Yang prepared the manuscript for publication and reviewed the draft. All authors read and approved the final manuscript.

\section{References}

1. Ayanlowo 0, Akinkugbe A. Clinical pattern of psoriasis in patients seen at a tertiary hospital in Nigeria. J Clin Sci. 2016;13(3):137-42. https://doi.org/10.4103/2468-6859.185251

2. Kim J, Krueger JG. The immunopathogenesis of psoriasis. Dermatol Clin. 2015;33(1):13-23. https://doi.org/10.1016/j. det.2014.09.002

3. Zhang $Y$, Tu C, Zhang D, Zheng Y, Peng Z, Feng Y, et al. Wnt/ $\beta$-catenin and $\mathrm{Wnt} 5 \mathrm{a} / \mathrm{Ca}$ pathways regulate proliferation and apoptosis of keratinocytes in psoriasis lesions. Cell Physiol Biochem. 2015;36:1890-902. https://doi. org/10.1159/000430158 
4. Lulevich V, Yang H-y, Rivkah Isseroff R, Liu G-y. Single cell mechanics of keratinocyte cells. Ultramicroscopy. 2010; 110(12): 1435-42. https://doi.org/10.1016/j.ultramic.2010.07.009

5. Hawkes JE, Chan TC, Krueger JG. Psoriasis pathogenesis and the development of novel targeted immune therapies. J Allergy Clin Immunol. 2017;140(3):645-53. https://doi. org/10.1016/j.jaci.2017.07.004

6. Zhang W, Yi X, An Y, Guo S, Li S, Song P, et al. MicroRNA-17-92 cluster promotes the proliferation and the chemokine production of keratinocytes: Implication for the pathogenesis of psoriasis. Cell Death Dis. 2018;9(5):567-567. https://doi. org/10.1038/s41419-018-0621-y

7. Gupta R, Ahn R, Lai K, Mullins E, Debbaneh M, Dimon M, et al. Landscape of long noncoding RNAs in psoriatic and healthy skin. J Invest Dermatol. 2016;136(3):603-9. https://doi. org/10.1016/j.jid.2015.12.009

8. Song J-K, Yin S-Y, Li W, Li X-D, Luo Y, Luo Y, et al. An update on the role of long non-coding RNAs in psoriasis. Chinese Med J. 2021;134(4):379-383. https://doi.org/10.1097/ CM9.0000000000001243

9. Li D, Kular L, Vij M, Herter EK, Li X, Wang A, et al. Human skin long noncoding RNA WAKMAR1 regulates wound healing by enhancing keratinocyte migration. Proc Nat Acad Sci. 2019;116(19):9443-52. https://doi.org/10.1073/pnas. 1814097116

10. Jia H-Y, Zhang K, Lu W-J, Xu G-W, Zhang J-F, Tang Z-L. LnCRNA MEG3 influences the proliferation and apoptosis of psoriasis epidermal cells by targeting miR-21/caspase- 8 . BMC Mol Cell Biol. 2019;20(1):46-46. https://doi.org/10.1186/ s12860-019-0229-9

11. Yao $\mathrm{Y}$, Wang $\mathrm{X}$, Gao J. LncRNA KCNQ10T1 sponges miR-206 to ameliorate neural injury induced by anesthesia via up-regulating BDNF. Drug Des Devel Ther. 2020;14:4789-800. https:// doi.org/10.2147/DDDT.S256319

12. Gao X, Ge J, Li W, Zhou W, Xu L. LncRNA KCNQ10T1 promotes osteogenic differentiation to relieve osteolysis via Wnt/ $\beta$ catenin activation. Cell Biosci. 2018;8(1):19. https://doi. org/10.1186/s13578-018-0216-4

13. Lin Z-B, Long $P$, Zhao Z, Zhang $Y-R$, Chu X-D, Zhao X-X, et al. Long noncoding RNA KCNQ10T1 is a prognostic biomarker and mediates CD8\&lt;sup\&gt;+\&lt;/sup\&gt; $T$ cell exhaustion by regulating CD155 expression in colorectal cancer [Internet]. Int J Biol Sci. 2021;17(7):1757-68. Available from: http://europepmc.org/abstract/MED/33994860; https://doi. org/10.7150/ijbs.59001; https://europepmc.org/articles/ PMC8120463; https://europepmc.org/articles/PMC8120463?pdf= render

14. Zhu Y, Shen $Y$, Chen R, Li H, Wu Y, Zhang F, et al. KCNQ10T1 IncRNA affects the proliferation, apoptosis, and chemoresistance of small cell lung cancer cells via the JAK2/STAT3 axis. Ann Transl Med. 2021;9(10):891-891. https://doi.org/10.21037/atm-21-1761

15. Shen $Y, X u$ J, Pan X, Zhang $Y$, Weng $Y$, Zhou D, et al. LncRNA KCNQ10T1 sponges miR-34c-5p to promote osteosarcoma growth via ALDOA enhanced aerobic glycolysis. Cell Death Dis. 2020;11(4):278-278. https://doi.org/10.1038/s41419-020-2485-1

16. Jiang X, Yu M, Zhu T, Lou L, Chen X, Li Q, et al. Kenq1ot1/miR$381-3 p / E T S 2$ axis regulates inflammation in mouse models of acute respiratory distress syndrome. Mol Ther Nucleic Acids. 2020;19:179-89. https://doi.org/10.1016/j.omtn.2019.10.036

17. Chen L, Xiong $Y$, Yan $C$, Zhou W, Endo $Y$, Xue H, et al. LncRNA KCNQ10T1 accelerates fracture healing via modulating miR701-3p/FGFR3 axis. FASEB J. 2020;34(4):5208-22. https://doi. org/10.1096/fj.201901864RR

18. Huang Y. The novel regulatory role of IncRNA-miRNAmRNA axis in cardiovascular diseases. J Cell Mol Med. 2018;22(12):5768-75. https://doi.org/10.1111/jcmm.13866

19. Li J, Tong $Y$, Zhou $Y$, Han Z, Wang $X$, Ding $T$, et al. LncRNA KCNQ10T1 as a miR-26a-5p sponge regulates ATG12-mediated cardiomyocyte autophagy and aggravates myocardial infarction. Intern J Cardiol. 2021;388:14-23. https://doi.org/10.1016/ j.ijcard.2021.05.053

20. Ling $\mathrm{C}$, Hu X, Luo L, Liang $\mathrm{C}$, Wang $\mathrm{H}$, Chen C. Phoenixin-14 regulates proliferation and apoptosis of vascular smooth muscle cells by modulation of KCNQ10T1/miR-183-3p/CTNNB1 axis. Environ Toxicol Pharmacol. 2021;86:103655. https://doi. org/10.1016/j.etap.2021.103655

21. Liu T, Zhang X, Wang Y. miR-183-3p suppresses proliferation and migration of keratinocyte in psoriasis by inhibiting GAB1. Hereditas. 2020;157(1):28-28. https://doi. org/10.1186/s41065-019-0104-x; https://doi.org/10.1186/ s41065-020-00138-w

22. Choi DH, Hwang HS. Anti-inflammation activity of Brazilin in TNF- $\alpha$ induced human psoriasis dermatitis skin model. Appl Biol Chem. 2019;62(1):46. https://doi.org/10.1186/ s13765-019-0455-z

23. Rendon A, Schäkel K. Psoriasis pathogenesis and treatment. Int J Mol Sci. 2019;20(6):1475. https://doi.org/10.3390/ ijms20061475

24. Li Y, Shi B, Dong F, Zhu X, Liu B, Liu Y. LncRNA KCNQ10T1 facilitates the progression of bladder cancer by targeting MiR-218-5p/HS3ST3B1. Cancer Gene Ther. 2021;28(3):212-20. https://doi.org/10.1038/s41417-020-00211-6

25. Benhadou F, Mintoff D, del Marmol V. Psoriasis: Keratinocytes or immune cells - Which is the trigger? Dermatology. 2019;235(2):91-100. https://doi.org/10.1159/000495291

26. Ru Y, Li H, Zhang R, Luo Y, Song J, Kuai L, et al. Role of keratinocytes and immune cells in the anti-inflammatory effects of tripterygium wilfordii hook. $f$. in a murine model of psoriasis. Phytomedicine. 2020;77:153299. https://doi.org/10.1016/j. phymed.2020.153299

27. He Q, Liu N, Hu F, Shi Q, Pi X, Chen H, et al. Circ_0061012 contributes to IL-22-induced proliferation, migration and invasion in keratinocytes through miR-194-5p/GAB1 axis in psoriasis. Biosci Rep. 2021;41(1):BSR20203130. 